\title{
NEAR RINGS CHARACTERIZED BY INTUITIONISTIC FUZZY BI IDEALS
}

\author{
K. Dhilipkumar \\ Department of Mathematics \\ SSM College of Arts and Science \\ Komarapalayam - 638 183, Tamilnadu, India \\ e-mail: dhilipkumarmaths@gmail.com \\ AND \\ M. RAMACHANDRAN \\ Department of Mathematics \\ Government Arts and Science College \\ Sathyamangalam - 638 401, Tamilnadu, India \\ e-mail: ramachandran64@gmail.com
}

\begin{abstract}
Generalizing the concept of fuzzy bi ideals of near rings, the notion of intuitionistic fuzzy bi ideals of near rings is introduced. We also characterize and investigate some related properties of intuitionistic fuzzy bi ideals of near rings.

Keywords: near rings, fuzzy bi ideals, intuitionistic fuzzy subnear ring, intuitionistic fuzzy ideals, intuitionistic fuzzy bi ideals.
\end{abstract}

2010 Mathematics Subject Classification: 16D25, 03E72, 03F55, 16 Y30.

\section{REFERENCES}

[1] S. Abou-Zaid, On fuzzy sub near rings and ideals, Fuzzy Sets and Systems 44 (1991) 139-146. doi:10.1016/0165-0114(91)90039-S

[2] K.T. Atanassov, Intuitionistic fuzzy sets, Fuzzy Sets and Systems 20 (1986) 87-96. doi:10.1016/S0165-0114(86)80034-3

[3] T.T. Chelvam and N. Ganesan, On bi ideals of near rings, Indian J. Pure Appl. Math. 18 (1987) 1002-1005. 
[4] Z. Jianming and M. Xueling, Intuitionistic fuzzy ideals of near rings, Scientae Math. Japonicae 61 (2004) 219-223.

[5] Y.B. Jun, Intuitionistic fuzzy bi-ideals of ordered semigroups, Kyungpook Math. J. 45 (2005) 527-537.

[6] N. Kuroki, Fuzzy bi ideals in semigroups, Comment. Math. Univ. St. Pauli. 28 (1979) 17-21.

[7] N. Kuroki, On fuzzy ideals and fuzzy bi ideals in semigroups, Fuzzy Sets and Systems 5 (1981) 203-215. doi:10.1016/0165-0114(81)90018-X

[8] N. Kuroki, Fuzzy semiprime bi ideals in semigroups, Fuzzy Sets and Systems 8 (1981) $71-79$ doi:10.1016/0165-0114(82)90031-8

[9] W. Liu, Fuzzy invariants subgroups and fuzzy ideals, Fuzzy Sets and Systems 8 (1982) 133-139. doi:10.1016/0165-0114(82)90003-3

[10] T. Manikantan, Fuzzy bi ideals of near rings, J. Fuzzy Math. 17 (2009) 1-13.

[11] R.G. McLean and H. Kummer, Fuzzy ideals in semigroups, Fuzzy Sets and Systems 48 (1992) 137-140. doi:10.1016/0165-0114(92)90258-6

[12] G. Pilz, Near Rings (North Holland, Amsterdam, 1983).

[13] A. Rosenfeld, Fuzzy groups, J. Math. Anal. Appl. 35 (1971) 512-517. doi:10.1016/0022-247X(71)90199-5

[14] P.K. Sharma, Intuitionistic ideal of a near rings, Int. Math. Forum 7 (2012) 769-776.

[15] I. Yakabe, Quasi ideals in near rings, Math. Rep. XIV-1 (1983) 41-46.

[16] L.A. Zadeh, Fuzzy sets, Inform. and Control 8 (1965) 338-353. doi:10.1016/S0019-9958(65)90241-X

Received 4 July 2019

Revised 24 August 2019

Accepted 24 March 2020 